\title{
Evaluation of a Solid-State Gyroscope for Robotics Applications
}

\author{
Billur Barshan and Hugh F. Durrant-Whyte, Member, IEEE
}

\begin{abstract}
The evaluation of a low-cost solid-state gyroscope for robotics applications is described. An error model for the sensor is generated and included in a Kalman filter for estimating the orientation of a moving robot vehicle. Orientation estimation with the error model is compared to the performance when the error model is excluded from the system. The results demonstrate that without error compensation, the error in localization is between $5-15^{\circ} / \mathrm{min}$ but can be improved at least by a factor of 5 if an adequate error model is supplied. Like all inertial systems, the platform requires additional information from some absolute position-sensing mechanism to overcome long-term drift. However, the results show that with careful and detailed modeling of error sources, inertial sensors can provide valuable orientation information for mobile robot applications.
\end{abstract}

\section{INTRODUCTION}

TERTIAL navigation systems are self-contained, nonradiating, nonjammable, dead-reckoning navigation systems which provide dynamic information through direct measurements. In most cases an INS must be integrated with other absolute location-sensing mechanisms to provide useful information about vehicle position. Models that describe the outputs of inertial sensors sufficiently accurately are essential if the information is to be used effectively. Fundamentally, gyros provide angular rate information, and accelerometers provide velocity rate information. Although the rate information is reliable over long periods of time, it must be integrated to provide absolute measurements of orientation, position and velocity. Thus, even very small errors in the rate information provided by inertial sensors cause an unbounded growth in the error of integrated measurements. As a consequence, an INS by itself is characterized by position errors that grow with time and distance. One way of overcoming this problem is to periodically reset inertial sensors with other absolute sensing mechanisms and so eliminate this accumulated error. In robotics applications, a number of systems have been described which use some form of absolute sensing mechanisms for guidance (see [1] or [2] for surveys). Such systems typically rely on the availability of easy-to-see beacons or landmarks, using simple encoder information to predict vehicle location between sensing locations. This works well when the density of beacons or landmarks is high and the ground over which the vehicle travels is relatively smooth. In cases

Manuscript received October 6, 1993; revised July 6, 1994.This work was supported in part by SERC-ACME under Grant GR/6 38375 .

B. Barshan is with Department of Electrical Engineering Bilkent University Bilkent, TR-06 533 Ankara, Turkey.

H. F. Durrant-Whyte is with the Robotics Research Group Department of Engineering Science University of Oxford Oxford, OX1 3PJ, UK. IEEE Log Number 9408146. where the beacon density is sparse, such systems can easily lose position track. Inertial navigation systems can potentially overcome this problem. Inertial information can be used to generate estimates of position over significant periods of time, independent of landmark visibility and of the validity of encoder information. Clearly, positions derived from inertial information must occasionally be realigned using landmark information, but a system that combines both inertial and landmark sensing can cope with substantially lower landmark density and can also deal with rough terrain where encoder information has limited value.

Inertial navigation systems have been widely used in aerospace applications [3], [4] but have yet to be seriously exploited in robotics applications where they have considerable potential. In [5], the integration of inertial and visual information is investigated. Methods of extracting the motion and orientation of the robotic system from inertial information are derived theoretically but not directly implemented as a real system. In [6], inertial sensors are used to estimate the attitude of a mobile robot. With the classical three-gyro, twoaccelerometer configuration used in aeronautics, experiments are performed to estimate the roll and pitch of the robot when one wheel climbs onto a plank using a small inclined plane. In [7], a rate gyroscope is used to lock the heading of a semiautonomous mobile robot to a prespecified direction. Here, the bias of the gyroscope is measured when the robot is not in motion, and it is assumed to be constant throughout the experiments. More sophisticated error modeling is necessary to reduce and compensate for the drift error of low-cost rate gyroscopes.

One reason that inertial systems are widely used in aerospace applications but not in robotics applications is simply that high-quality aerospace inertial systems are comparatively too expensive for most robotics systems. However, low-cost solid-state inertial systems, motivated by the needs of the automotive industry, are increasingly being made commercially available. Although a considerable improvement on past systems, they clearly provide substantially less accurate position information than equivalent aerospace systems. However, as we describe in this paper, such systems are at a point that, by developing reasonably detailed models of the sensors involved, they can provide valuable information in many robot positioning tasks.

To make best use of low-cost inertial sensing systems, it is important that a detailed understanding of the mechanisms causing drift error are understood and a model for these derived. The approach taken in this paper is to incorporate in 


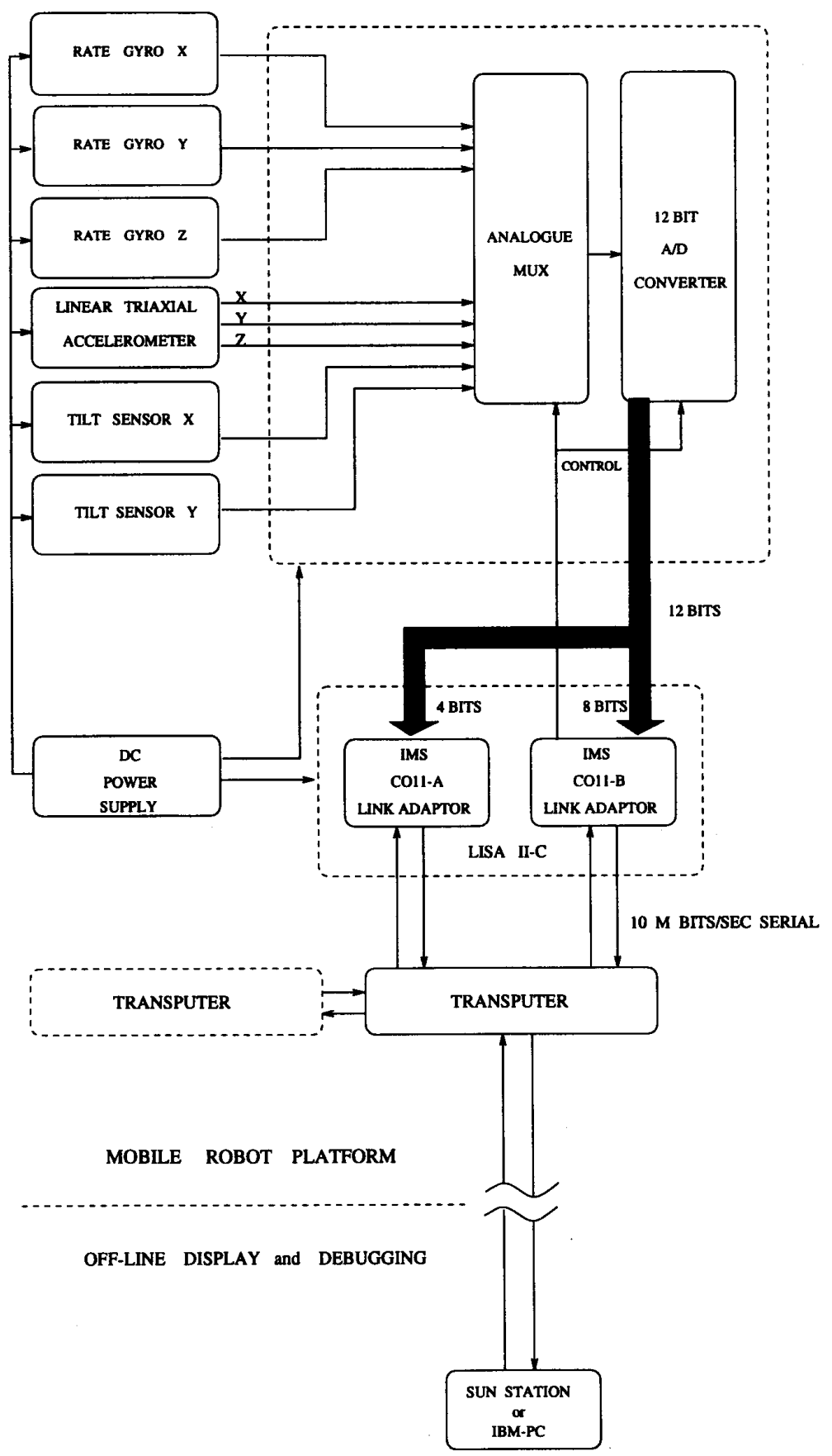

Fig. 1. Hardware implementation of the INS.

the system a priori information about the error characteristics of the inertial sensors and to use this directly in a Kalman Filter (KF) to estimate position before supplementing the gyroscope with absolute sensing mechanisms. Section II introduces the gyroscope being evaluated and briefly describes the hardware configuration. Section III focuses on generating an error model for the gyroscope and testing it for adequacy of representation.
In Section IV, the error model is exploited in a KF to compensate for the error. The system performance with the error model is compared to the performance with no error model in Section V, and a statement of accuracy is included. In the concluding Section VI, the usefulness of low-cost gyroscopes in robotics applications is discussed for guidance systems for mobile robots. 


\section{IMPLEMENTATION OF THE INS}

A fundamental requirement for an autonomous mobile robot is the ability to localize itself with respect to the environment. In the Oxford AGV laboratory, an INS is under current development to aid in accomplishing various robotic tasks such as localization and target tracking in a 2-D environment. The system comprises a Solid STate Angular Rate Transducer (START) gyroscope (approximate cost: $£$ 1500), a linear triaxial accelerometer, and two Electrolevel inclinometers. The block diagram for the hardware implementation of the inertial sensors is shown in Fig. 1.

The START gyroscope is an inertial sensor originally intended for the guided munition market in the 1980's but which has also proved to be very suitable for the vehicle control market [8], [9]. The device consists of a small cylinder with integral piezoelectric transducers and an integrated-circuit module [10]. The principal of operation is to measure the Coriolis acceleration caused by angular rotation of a vibrating cylinder, chosen for its symmetry, around the principal axis. The cylinder is open at one end and supported on a base at the other end. Eight piezoelectric transducers are attached symmetrically around the open end of the cylinder for driving, controlling and measuring the vibrations via the integratedcircuit module [11].

The gyroscope generates a voltage output proportional to the angular velocity of the vehicle around the principle axis of the device. The maximum rate that can be measured with the particular START gyro under investigation is $\pm 200^{\circ} / \mathrm{s}$ within its linear range. If the input rate goes beyond the maximum limits, the rate and orientation information become erroneous and need to be reset.

The angular rate from the gyroscope is multiplexed with information from other inertial sensors in the system and fed to a 12-bit $A / D$ converter. The digitized output is interfaced to an INMOS-T805 transputer network.

\section{CONSTRUCting AN ERror Model For The Gyroscope}

Building error models for inertial sensors is motivated by an attempt to reduce the effect of unbounded position and orientation errors. Depending on how successful these models are, inertial sensors may possibly be used in an unaided mode or for longer durations on their own. The gyroscope drift in its various manifestations is the most important contributor to navigation system errors, and is mainly dependent upon the device technology. A detailed treatment of modeling aerospace INS's can be found in the first volume in [12]. For a robotic INS, the scale, nature and parameters of the localization problem are different than in aerospace. Hence, INS's developed for aerospace applications cannot be directly implemented on mobile ground vehicles. In addition, systems developed for aerospace are far too expensive to be used in robotics applications.

To develop an error model for the gyroscope under investigation, its output was recorded over long periods of time when subjected to zero input, i.e., the gyroscope was stationary on the laboratory bench. The experiments were performed overnight when the vibrations in the building were

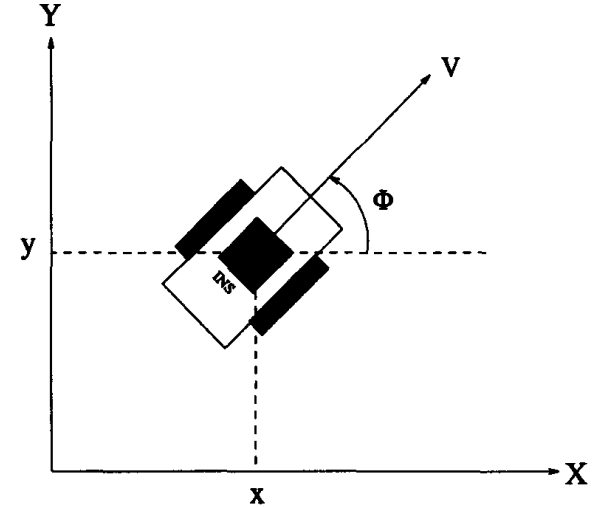

Fig. 2. The mobile robot equipped with the gyro.

at a minimum. The result of sample experiments over a period of 12 hours is shown in Fig. 3 for START. Ideally, the output for zero input would be a constant voltage level corresponding to the digital output of 2048 for a 12-bit A/D converter as shown by the thick, solid horizontal line in the figure. The standard deviation of the output fluctuations is approximately $0.16 \%$ s. The real output data is at a lower level than ideal at start-up, and the mean value gradually increases with time in an exponential fashion. Repeatability of these results indicates that an apparently small time-varying bias is characteristic of this gyroscope. Although a single specimen of START has been available for the experiments, three specimens of another solid-state gyro have also been shown to have the same type of exponential error model with different parameter values [13]. The time variation of the bias is attributed to thermal effects based on the observation that the device gradually heats up during operation. The bias can taper off to a negative or positive value depending on the ambient temperature.

In the following, let $\epsilon(t)$ be the bias error associated with measuring the angular rate of rotation using the START. A nonlinear parametric model of the following form was fitted to the data from the gyroscope using the Levenberg-Marquardt iterative least-squares fit method [14]:

$$
\epsilon_{\text {model }}(t)=C_{1}\left(1-e^{-\frac{t}{T}}\right)+C_{2}
$$

where $\epsilon$ model $(t)$ is the fitted error model to the gyroscope output when zero input was applied, with parameters $C_{1}, C_{2}$ and $T$ to be tuned. Starting with reasonable initial guesses for the parameters, convergence to a local minimum is achieved within 5-10 iterations. The best fitting parameter values to the experimental data are $C_{1}=2.3 \mathrm{~A} / \mathrm{D}$ units (or $0.300^{\circ} / \mathrm{sec}$ ), $C_{2}=2045.5 \mathrm{~A} / \mathrm{D}$ units (or $-0.326^{\circ} / \mathrm{sec}$ ) and $T_{1}=1.06 \mathrm{hr}$ (or $63.6 \mathrm{~min}$ ) with the corresponding fitted curve shown in the thin solid line in Fig. 3.

In general, a model fitted to experimental data is regarded as being adequate if the residuals from the fitted model constitute a white, zero-mean process. Hence, one can start with any reasonable model based on inspecting the original data and develop it further if necessary until the residuals are white. Following this route, the sufficiency of the above model in Equation 1 is determined for the gyroscope by applying a whiteness test to the residuals in the autocorrelation domain. 


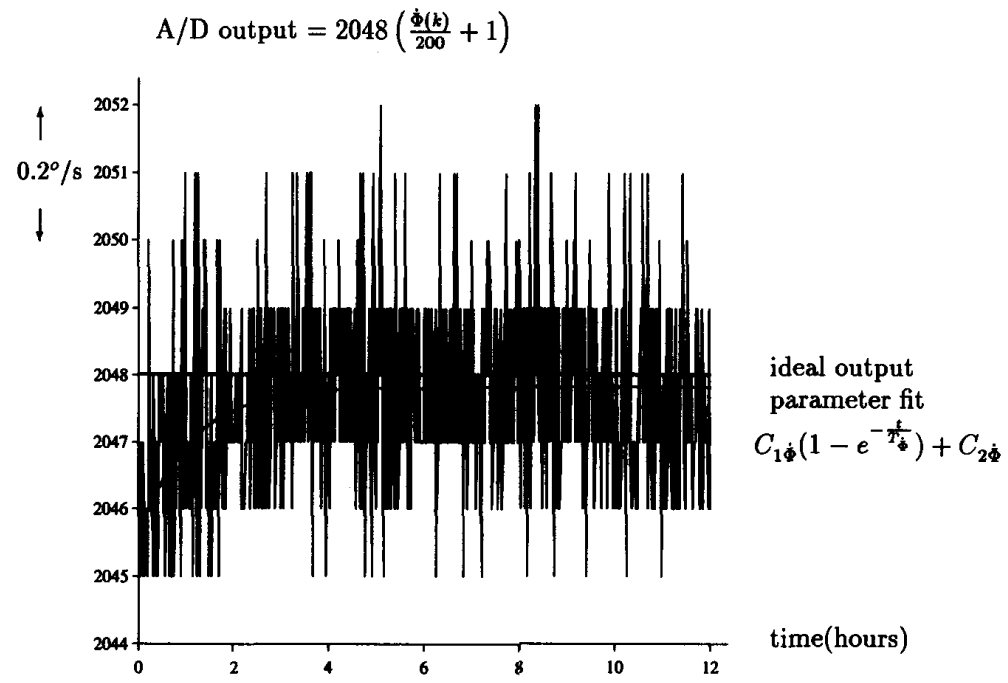

Fig. 3. Digitized angular rate output of the START gyroscope when subjected to zero input. Data was collected over a period of 12 hours by sampling every minute when no angular rotation was applied around the principal axis.

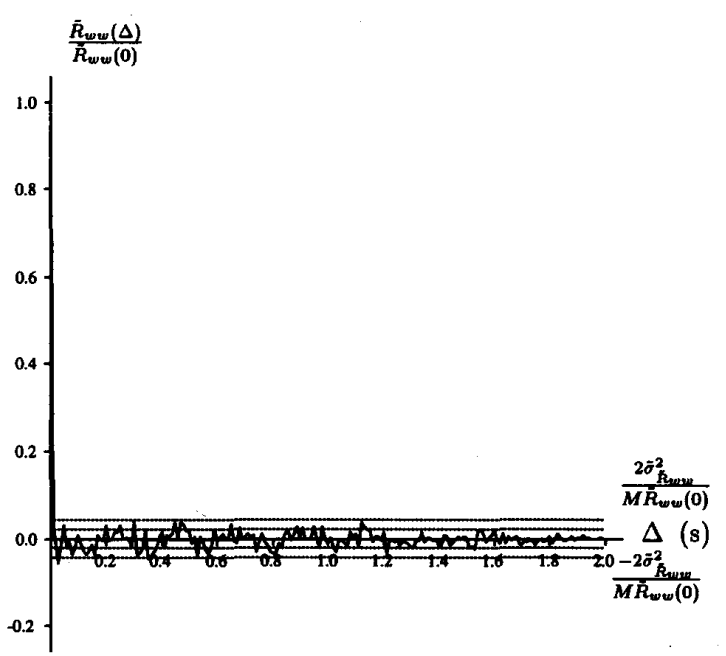

Fig. 4. Biased sample autocorrelation estimate of the residuals. The result was obtained by an ensemble average over the autocorrelations of 10 data sequences, each of $10 \mathrm{~s}$ duration. The dotted lines indicate $\frac{ \pm \tilde{\sigma}_{\tilde{R}_{w w}}}{\sqrt{M} \tilde{R}_{w w}(0)}$ and $\frac{ \pm 2 \tilde{\sigma}_{\bar{R}_{w w}}}{\sqrt{M} \tilde{R}_{w w}(0)}$ bounds for the autocorrelation estimate.

For a discrete system with sampling interval $T_{s}$, the residual $w(k)$ at time $k T_{s}$ is computed as follows:

$$
w(k)=\epsilon(k)-\epsilon \text { model }(k) .
$$

Since the process $w(k)$ is assumed to be stationary, the autocovariance $R_{w w}$ is only a function of the lag $\Delta$ between two data samples. When only a finite set of $N$ data samples is available for estimation, the expression for the sample biased autocovariance estimate is given by [15]

$$
\tilde{R}_{w w}(\Delta)=\frac{1}{N} \sum_{k=0}^{N-|\Delta|-1} w(k) w(k+\Delta) .
$$

If $N$ is sufficiently large $(N \geq 16)$, it can be shown that [16] the distribution of the sample autocovariance estimate for nonzero $\Delta$ is well approximated by a Gaussian distribution with zero mean and standard error given by

$$
\tilde{\sigma}_{\tilde{R}_{w w}}(\Delta)=\frac{1}{\sqrt{N}} \tilde{R}_{w w}(0) \quad \text { for } \quad \Delta \neq 0 .
$$

In Fig. 4, the sample autocorrelation estimate is shown for the START. An ensemble average over the autocorrelation estimates of $M=10$ data sequences (each of $10 \mathrm{~s}$ duration) was taken, reducing the standard error bounds by $\frac{1}{\sqrt{M}}$. The dotted lines correspond to \pm 1 and \pm 2 standard error bounds for the autocorrelation estimate [17]. Since the sample autocorrelation error distribution of a white process is Gaussian, the autocorrelation estimate is bound to lie within \pm 1 standard error $95.5 \%$ of the time. In compliance, the results indicate that the estimate is within these limits about $96 \%$ of the time. The positive outcome of the whiteness test on the model residuals demonstrates that the model in (1) adequately represents the slowly varying bias error on the rate output of the START gyroscope. In the next section, the error models developed are exploited in a $\mathrm{KF}$ to compensate for the errors.

\section{IMPLEMENTATION OF THE GyrosCOPE ERROR MODEL}

The parametrized model of (1) for the bias error can be represented by the following differential equation:

$$
\dot{\epsilon}_{\dot{\Phi}}(t)=\frac{C_{1}+C_{2}}{T_{1}}-\frac{1}{T_{1}} \epsilon_{\dot{\Phi}}(t)
$$

with initial conditions $\epsilon_{\dot{\Phi}}(0)=C_{2}$ and $\dot{\epsilon}_{\dot{\Phi}}(0)=\frac{C_{1}}{T_{1}}$. After discretization using the backward difference method, (5) becomes

$$
\begin{gathered}
\epsilon_{\dot{\Phi}}(k+1)=\frac{T_{1}}{T_{1}+T_{s}} \epsilon_{\dot{\Phi}}(k)+\frac{T_{s}}{T_{1}+T_{s}}\left(C_{1}+C_{2}\right) \\
\text { with } \quad \epsilon_{\dot{\Phi}}(0)=C_{2} .
\end{gathered}
$$


The minimum possible $T_{s}$ of $30 \mathrm{~ms}$ was chosen to keep the discretization error small. Due to its recursive nature, this difference equation is independent of start-up time but relies on a good estimate of the initial bias.

The system observation is the erroneous rate output of the gyroscope which corresponds to the true value of the rate plus the bias error (see (7), shown at the bottom of the page)or in matrix notation:

$$
\mathbf{z}(k)=\mathbf{H} \boldsymbol{\Phi}(k)+\mathbf{v}(k)
$$

where $v(k)$ is a white measurement noise process.

Given the erroneous observations, the states that need to be estimated are the true values of orientation, angular rate and the errors associated with them. Hence, the state equations of a linear KF are augmented by (6) and its integral to estimate and compensate for the bias error. The resulting state equations of the filter are as follows (see (9), shown at the bottom of the page) or in matrix notation:

$$
\boldsymbol{\Phi}(k+1)=\mathbf{F} \boldsymbol{\Phi}(k)+\mathbf{u}+\mathbf{w}(k) .
$$

The first four states are the true values of the orientation and its derivatives, and the last two states constitute the error model for the gyroscope. Note that the error states are coupled to the relevant true states only through the observation and not by the structure of the state transition matrix $\mathbf{F}$. In setting the covariance matrix $\mathbf{Q}$ for the process noise $\mathbf{w}(k)$, a continuous-time white-noise model assumption has been made as described in [18]. This assumption leads to the following process covariance matrix in discrete time:

$$
\begin{aligned}
& \mathbf{Q}=E\left\{\mathbf{w}(k) \mathbf{w}^{\mathbf{T}}(k)\right\}= \\
& \left.\qquad \begin{array}{cccccc}
\frac{T_{s}^{7}}{252} \sigma_{u}^{2} & \frac{T_{s}^{6}}{72} \sigma_{u}^{2} & \frac{T_{s}^{5}}{30} \sigma_{u}^{2} & \frac{T_{s}^{4}}{24} \sigma_{u}^{2} & 0 & 0 \\
\frac{T_{s}^{6}}{72} \sigma_{u}^{2} & \frac{T_{s}^{5}}{20} \sigma_{u}^{2} & \frac{T_{s}^{4}}{8} \sigma_{u}^{2} & \frac{T_{s}^{3}}{6} \sigma_{u}^{2} & 0 & 0 \\
\frac{T_{s}^{5}}{30} \sigma_{u}^{2} & \frac{T_{s}^{4}}{8} \sigma_{u}^{2} & \frac{T_{s}^{3}}{3} \sigma_{u}^{2} & \frac{T_{s}^{2}}{2} \sigma_{u}^{2} & 0 & 0 \\
\frac{T_{s}^{4}}{24} \sigma_{u}^{2} & \frac{T_{s}^{3}}{6} \sigma_{u}^{2} & \frac{T_{s}^{2}}{2} \sigma_{u}^{2} & T_{s} \sigma_{u}^{2} & 0 & 0 \\
0 & 0 & 0 & 0 & \frac{T_{s}^{3}}{3} \sigma_{w}^{2} & \frac{T_{s}^{2}}{2} \sigma_{w}^{2} \\
0 & 0 & 0 & 0 & \frac{T_{s}^{2}}{2} \sigma_{w}^{2} & T_{s} \sigma_{w}^{2}
\end{array}\right]
\end{aligned}
$$

where $\sigma_{u}=0.05^{\circ} / \mathrm{sec}^{3}$, and the $\sigma_{w}$ is the standard deviation of the residuals from the fitted model experimentally determined to be $2.14 \mathrm{~A} / \mathrm{D}$ units or $0.2^{\circ} / \mathrm{sec}$. The state vector estimated by the filter is given by

$$
\hat{\mathbf{\Phi}}(k+1 \mid k+1)=\mathbf{F} \hat{\mathbf{\Phi}}(k \mid k)+\mathbf{u}+\mathbf{W}(k+1) \nu(k+1)
$$

where $\mathbf{W}(k+1)$ is the filter gain, and $\nu(k+1)=\mathbf{z}(k+$ 1) $-\mathbf{H} \hat{\boldsymbol{\Phi}}(k+1 \mid k)$ is the innovations vector provided by the new observation at time $(k+1) T_{s}$. A detailed treatment of $\mathrm{KF}$ prediction and update equations can be found in [18].

The above filter has a constant $\dddot{\Phi}(k)$ structure augmented by the error model. Lower-order filters have been implemented but shown to have a delay and much ringing in their unit-step response. With this higher-order model, the filter is able to track abrupt changes in angular velocity very closely as will be shown in the next section.

The filter structure in (7) and (9) has been implemented in real time on an INMOS-T805 transputer network where a minimum sampling interval of $T_{s}=50 \mathrm{~ms}$ is achieved. The START gyroscope has been mounted on a rotating platform whose angular velocity and orientation can be accurately controlled and measured. A 500-line optical encoder was used to measure motor shaft position, driving the platform through a low backlash 40:1 gear box. An HCTL-1 100 chip was used to control the motor in the integral velocity mode. The motor position from the encoder is accurate to $1 / 2000$ of a revolution. The most significant positioning error is in gear-box backlash. This is very good, however, and better than $1 / 10$ of a degree. For comparison purposes, the platform velocity and orientation are taken to be the "true" values of these quantities in the next section. An initial estimate of the bias, $\epsilon_{\dot{\Phi}}(0)$, is made to initialize the filter by averaging the output of the gyroscope over a large number of samples when there is no rotational motion. Since the start time of the experiment can correspond to any point on the curve in Fig. 3, it is important to have a good estimate of the initial bias. For an initial estimate with over 2000 samples, data collection and estimation take only 1-2 sec on an INMOS-T805 transputer hosted by an IBM-80 486 PC. As data is collected by the gyroscope, the $\mathrm{KF}$ process running in parallel filters the measurements and provides estimates of orientation, its derivatives and the state of the bias error.

$$
\begin{gathered}
{\left[\dot{\Phi}(k)+\epsilon_{\dot{\Phi}}(k)+v(k)\right]=\left[\begin{array}{llllll}
0 & 1 & 0 & 0 & 0 & 1
\end{array}\right]\left[\begin{array}{c}
\Phi(k) \\
\dot{\Phi}(k) \\
\ddot{\Phi}(k) \\
\dddot{\Phi}(k) \\
\epsilon_{\Phi}(k) \\
\epsilon_{\dot{\Phi}}(k)
\end{array}\right]+[v(k)]} \\
{\left[\begin{array}{c}
\Phi(k+1) \\
\dot{\Phi}(k+1) \\
\ddot{\Phi}(k+1) \\
\dddot{\Phi}(k+1) \\
\epsilon_{\Phi}(k+1) \\
\epsilon_{\dot{\Phi}}(k+1)
\end{array}\right]=\left[\begin{array}{ccccccc}
1 & T_{s} & \frac{1}{2} T_{s}^{2} & \frac{1}{6} T_{s}^{3} & 0 & 0 \\
0 & 1 & T_{s} & \frac{1}{2} T_{s}^{2} & 0 & 0 \\
0 & 0 & 1 & T_{s} & 0 & 0 \\
0 & 0 & 0 & 1 & 0 & 0 \\
0 & 0 & 0 & 0 & 1 & T_{s} \\
0 & 0 & 0 & 0 & 0 & \frac{T_{1}}{T_{1}+T_{s}}
\end{array}\right]\left[\begin{array}{c}
\Phi(k) \\
\dot{\Phi}(k) \\
\ddot{\Phi}(k) \\
\dddot{\Phi}(k) \\
\epsilon_{\Phi}(k) \\
\epsilon_{\dot{\Phi}}(k)
\end{array}\right]+\left[\begin{array}{c}
0 \\
0 \\
0 \\
0 \\
0 \\
\frac{T_{s}\left(C_{1}+C_{2}\right)}{T_{1}+T_{s}}
\end{array}\right]+\left[\begin{array}{c}
0 \\
0 \\
0 \\
u(k) \\
0 \\
w(k)
\end{array}\right]}
\end{gathered}
$$



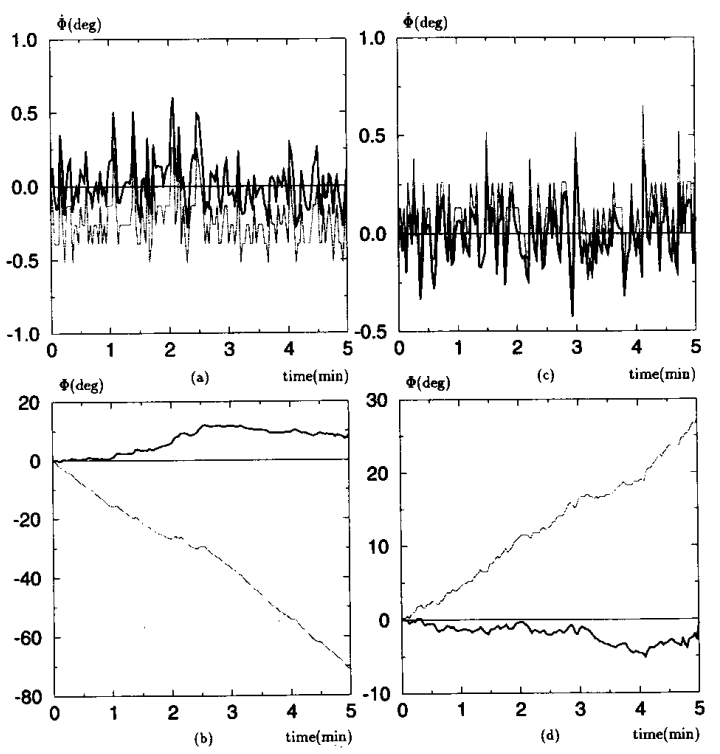

Fig. 5. On the left, angular rate (top) and orientation (bottom) for the zero-input case of the START gyro when the bias error is negative. On the right, angular rate (top) and orientation (bottom) when the bias error is positive. The true values (thin, solid line) and the erroneous observations (dotted line) are illustrated along with the KF output (heavy, solid line) which compensates for the error.

\section{Performance OF The Start Gyroscope}

To determine the adequacy of the error models, the system performance with no assumed error model is compared to the performance when the error model is incorporated in the $\mathrm{KF}$ for the gyroscope.

The results when zero input was applied to the START gyroscope are shown in Fig. 5 over a duration of five minutes. The true values and the erroneous observations are illustrated along with the KF output which compensates for the error. In Fig. 5(a) and (b), the system was close to start-up, and the bias error had negative values. At the end of the experiment, the integrated gyroscope rate output exhibited a worst-case error of $-70.8^{\circ} / \mathrm{s}$, whereas the compensated and filtered output was $+8.6^{\circ}$, having had an overall maximum deviation of $+12.0^{\circ}$ from the true value. In Fig. 5(c) and (d), the system had warmed up, and the bias had achieved a positive value. At the end of the experiment, the integrated gyroscope rate output was $+27.4^{\circ} / \mathrm{s}$ corresponding to the worst case, whereas the compensated and filtered output was $-0.6^{\circ}$, having had an overall maximum deviation of $-5.1^{\circ}$ from the true value. In both cases, the improvement was approximately a factor of 6 .

Fig. 6 illustrates the angular rate and position of the START gyroscope when nonzero input was applied for a total duration of five minutes. A new angular rate $-25 \leq \dot{\Phi} \leq 25^{\circ} / \mathrm{s}$ was randomly generated every $30 \mathrm{~s}$ and applied to the gyro. The true values and the erroneous observations are displayed along with the filter results. Note that the drift in the orientation is more significant than in the angular rate since even very small errors quickly accumulate when integrated. To make this more visible, the true and estimated errors in rate and orientation
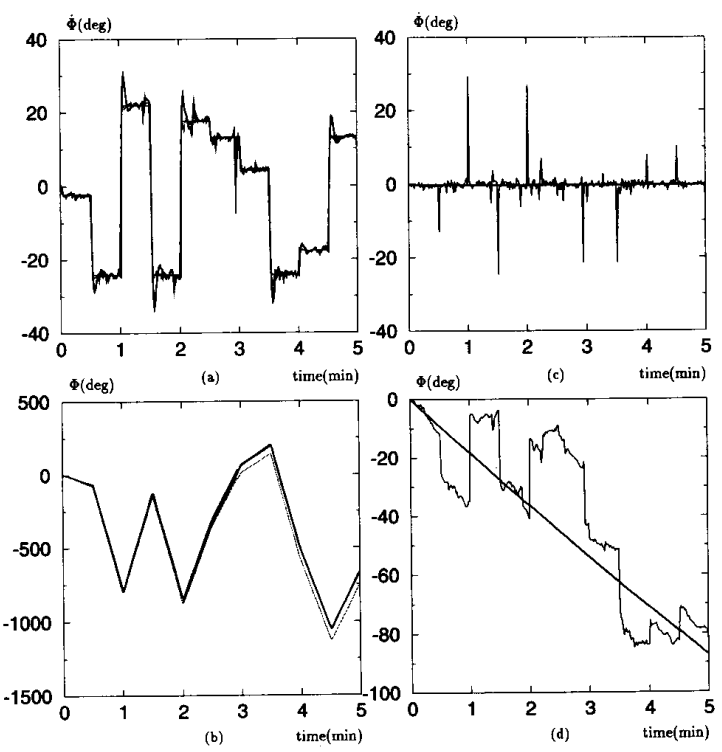

Fig. 6. On the left, angular rate (top) and position (bottom) of the START gyroscope when nonzero input was applied are shown. A new angular rate was randomly generated every $30 \mathrm{~s}$ and applied to the gyro. The true values (thin, solid lines) and the erroneous observations (dotted lines) are displayed along with the KF results (thick, solid lines) which compensate for the error. On the right, error in the angular rate (top) and error in orientation (bottom) are illustrated. Both the true (thin, solid lines) and the estimated values (heavy, solid lines) are shown.

are shown separately in Fig. 6(c) and (d) for the same data. At the end of the experiment, the integrated rate output was erroneous by $-84.7^{\circ}$ (the worst case) whereas the filtered estimate had an error of $+3.4^{\circ}$, indicating that the filter slightly over-compensated for the bias in this particular case. During the course of the experiment, however, the compensation was not always as good, the worst-case error being $36.0^{\circ}$, due to the large spiky errors in the measured angular rate at those points when a new rate was suddenly applied to the gyro. These errors can be seen in Fig. 6(d) more clearly. Both the gyroscope rate output and the filtered rate output were accurate within $\pm 2.5^{\circ} / \mathrm{s}$ at the end of the experiment.

\section{Discussion AND CONClusion}

The purpose of the research described in this paper was to evaluate a low-cost sensor for orientation estimation in mobile robot guidance problems. By developing a careful and accurate error model of the device, substantial improvements in performance can be made which make the application of lowcost inertial navigation systems to mobile robot applications a viable proposition.

The results demonstrate that the START gyroscope provides accurate localization only if an adequate error model is generated and supplied to the KF model. The model reduces the angular position error at least by a factor of 5 to 7 . With the resulting accuracy, it is still necessary to reset the error with absolute sensing information at regular intervals, approximately every 10 minutes. Since the filter provides an estimate of the error in orientation, one way of determining 
how often to reset the system is to threshold the absolute value of this estimate. The START gyroscope with the given model would operate particularly well in environments where the ambient temperature is controlled. Our experiments were performed in a room where the thermostat was set to $25^{\circ} \mathrm{C}$, but $\pm 2^{\circ}$ deviations were possible from the nominal value. Hence, the model sometimes over- or under-compensated for the errors.

The error model developed in this paper is valuable since it is shown to be applicable to specimens of another rate gyro as well as other inertial sensors with different parameter values [13]. The results of integrating a solid-state gyroscope with other inertial sensors, mentioned in Section II, and testing of the system on an autonomous land vehicle are presented in succeeding work [13].

\section{ACKNOWLEDGMENT}

The authors would like to thank GEC Avionics, Kent U.K., for lending a prototype START gyroscope unit for evaluation purposes.

\section{REFERENCES}

[1] I. J. Cox and G. T. Wilfong, Eds., Autonomous Robot Vehicles. New York: Springer-Verlag, 1990.

[2] J. J. Leonard and H. F. Durrant-Whyte, Directed Sonar Navigation. New York: Kluwer, 1992.

[3] C. T. Leondes, Ed., Theory and Applications of Kalman Filtering. : London: Technical Editing and Reproduction, 1970.

[4] D. A. Mackenzie, Inventing Accuracy: A Historical Sociology of Nuclear Missile Guidance. Cambridge, MA: MIT Press, 1990.

[5] T. Vieville and O. D. Faugeras, Cooperation of the Inertial and Visual Systems. Berlin, Springer-Verlag, 1990.

[6] J. Vaganay and M. J. Aldon, "Attitude estimation for a vehicle using inertial sensors," in preprints of the Ist IFAC International Workshop on Intelligent Autonomous Vehicles, Southampton, Hampshire, U.K., D. Charnley, Ed. New York: Pergamon, 1993, pp. 89-94.

[7] T. Högström, T. Hultberg, and A. Wernerson, "A semi-autonomous robot with rate gyro supported control and a video camera," in D. Charnley, Ed., preprints of the lst IFAC International Workshop on Intelligent Autonomous Vehicles, Southampton, Hampshire, U.K.New York: Pergamon, 1993, pp. 25-30.

[8] D. G. Harris, "START: A novel gyro for weapons guidance," in University of Stuttgart Gyro Symposium, Stuttgart, Germany, 1988.

[9] $\ldots$, "Initial trials results for image stabilisation using a rugged low noise gyroscope," Tech. Rep., Guidance Syst. Div., GEC Avionics, Rochester, Kent, UK, 1992.
[10] C. H. J. Fox, "Vibrating cylinder gyro-theory of operation and error analysis," in University of Stuttgart Gyro Symposium, Stuttgart, Germany, 1988.

[11] R. M. Langdon, "The vibrating cylinder gyro," Tech. Rep., The Marconi Review, 1982.

[12] P. S. Maybeck, Stochastic Models, Estimation, and Control, vols. 1-3. New York: Academic Press, 1979.

[13] B. Barshan and H. F. Durrant-Whyte, "Inertial navigation systems for mobile robots," Robotics Research Group, Oxford Univ., Aug. 1993, Tech. Rep., No. OUEL 1998/93, to appear in IEEE Trans. Robotics Automat.

[14] S. A. Teukolsky W. H. Press, B. P. Flannery, and W. T. Vetterling, Numerical Recipes in C. Cambridge, UK: Cambridge University Press, 1988 , pp. $540-547$

[15] M. Schwartz and L. Shaw, Signal Processing: Discrete Spectral Analysis, Detection, and Estimation. New York: McGraw-Hill, 1975.

[16] R. L. Anderson, "Distribution of the serial correlation coefficient," Ann. Mathemat. Stat., vol. 13, pp. 1-13, Mar. 1942.

[17] G. E. P. Box and G. M. Jenkins, Statistical Models for Forecasting and Control. San Francisco, CA: Holden-Day, 1976.

[18] Y. Bar-Shalom and T. E. Fortmann, Tracking and Data Association. New York: Academic Press, 1988.

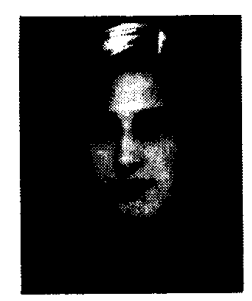

Billur Barshan received B.S. degrees in electrical engineering and physics from Bogaziçi University, Istanbul, Turkey, and the M.S. and Ph.D. degrees in electrical engineering from Yale University, New Haven, CT, in 1986, 1988, and 1991, respectively.

From 1987 to 1991, she was a Research Assistant at Yale University. From 1991 to 1993 , she was a Postdoctoral Researcher at the Robotics Research Group at University of Oxford, Oxford, U.K. Currently, she is an Assistant Professor at Bilkent University, Ankara, Turkey. Her current research interests include sensor-based robotics, sonar and inertial navigation systems and sensor data fusion.

Dr. Barshan is the recepient of the 1994 Nakamura Prize given to the most outstanding paper in IROS'93.

Hugh Durrant-Whyte (S'84-M'86) received the B.S. degree in nuclear and mechanical engineering from the University of London, England and the M.S.E.E. and Ph.D degrees from the University of Pennsylvania, Philadelphia in 1983,1985 , and 1986 respectively.

$\mathrm{He}$ is currently a University Lecturer in Engineering Science at the University of Oxford, England. His current research interests include multisensor systems and robotics. 